\title{
Linearly Extended $\pi$-Conjugated Dithiafulvene Polymer Formed Soluble Charge-Transfer Complex with 7,7,8,8-Tetracyanoquinodimethane
}

\author{
Kensuke NAKA, ${ }^{\dagger}$ Takashi UEMURA, and Yoshiki $\mathrm{CHUJO}^{\dagger}$ \\ Department of Polymer Chemistry, Graduate School of Engineering, Kyoto University, \\ Yoshida, Sakyo-ku, Kyoto 606-8501, Japan
}

(Received November 26, 1999)

\begin{abstract}
A soluble charge-transfer (CT) complex of $\pi$-conjugated donor polymer with 7,7,8,8-tetracyanoquinodimethane (TCNQ) was formed when TCNQ was added to a dimethyl sulfoxide (DMSO) solution of a $\pi$-conjugated poly(dithiafulvene) (2). In DMSO, 2 reacted with TCNQ to produce a dark green solution. After the precipitated TCNQ was filtered, the filtrate was evaporated to obtain a dark green powder. The resulting CT complex was soluble in acetonitrile, DMSO, $N, N$-dimethylformamide (DMF), acetone, and $\mathrm{MeOH}$, and partially soluble in tetrahydrofuran (THF). The UV-Vis absorption spectra suggested a formation of the CT complex containing about $1: 1$ ratio of dithiafulvene unit to TCNQ. The UV-Vis absorption and FT-IR analyses indicated that the anion radical of TCNQ was initially formed by the charge-transfer reaction when TCNQ was added to the solution of $\mathbf{2}$, and the remaining TCNQ was interacted with 2 as a partially charge-transferred form. In the ${ }^{1} \mathrm{H}$ NMR spectra, the broad peak attributed to the benzylidene proton of 2 was shifted to a lower magnetic field proportional to the TCNQ feed ratio against the dithiafulvene unit of 2 . The CT complex has a conductivity of $2 \times 10^{-4} \mathrm{~S} \mathrm{~cm}^{-1}$, three orders of magnitude greater than the uncomplexed polymer.

KEY WORDS Charge-Transfer Complex / Poly (dithiafulvene)/ $\pi$-Conjugated Polymer / Electrical Conductivity / 7,7,8,8,-Tetracyanoquinodimethane /
\end{abstract}

Derivatives of dithiafulvene, including tetrathiafulvalenes (TTF), act as electron donors and form stable charge-transfer (CT) complexes and radical ion salts with a wide range of acceptor species such as 7,7,8,8tetracyanoquinodimethane (TCNQ) ${ }^{1-3}$ The formation of CT complexes by chemical oxidation can be accomplished only if the redox potentials of the donors and acceptors are carefully balanced. The electrical conductivity of the solid state of TTF-TCNQ along the stacking direction is about three orders of magnitude higher than that in the perpendicular direction, and therefore TTFTCNQ behaves as a quasi-one-dimensional metal. Such low-dimensional conductors have been predicted by Peierls to undergo a lattice distortion at low temperatures resulting in a metal-insulator transition. ${ }^{1}$

In the poly-conjugated donor molecule, not only the processability of charge-transfer salts can be improved but the dimensionality of the conduction process can be increased in the doped state, due to the electron mobility along the polymer backbone via $\pi$-conjugation, as well as along the stacking direction via $\pi$-orbital overlap. ${ }^{4,5}$ There have been several attempts to incorporate dithiafulvene derivatives into the backbones of polymers, ${ }^{6-10}$ examples of polymers containing dithiafulvene units in the $\pi$-conjugated main chain have been limited. Müllen et al. have prepared a fully conjugated structure by inserting a TTF moiety in the conjugated backbone. ${ }^{11}$ However, due to its insolubility, the obtained material could not be characterized. Yamamoto et al. reported a preparation of soluble $\pi$-conjugated polymers containing TTF units in the $\pi$-conjugated main chain. ${ }^{12}$ These polymers did not form CT complexes with an organic acceptor such as TCNQ, however.

Recently, we reported a synthesis of a new $\pi$ conjugated polymer having electron-donating dithiaful-

\footnotetext{
${ }_{\text {t }}$ o whom correspondence should be addressed.
}

vene unit in the main chain by a cycloaddition polymerization of an aldothioketene derived from aromatic diyne monomer with its alkynethiol tautomer. ${ }^{13,14}$ The UV-Vis absorption spectra and cyclic voltammetry measurements showed an effective expansion of the $\pi$ conjugation system in the poly(dithiafulvene)s. In this paper, we report the first $\pi$-conjugated polymer containing dithiafulvene units in the main chain formed CT complex with TCNQ. The resulting CT complex was soluble in common organic solvents.

\section{EXPERIMENTAL}

\section{Materials}

Unless stated otherwise, all reagents and chemicals were obtained from commercial sources and used without further purification. Poly(dithiafulvene) (2) was prepared from 1,4-diethynylbenzene according to the previous our report. ${ }^{13,14}$ The number-average molecular weight $\left(M_{n}\right)$ of 2 used here was 3010 determined by ${ }^{1} \mathrm{H}$ NMR. Comparison of the intensities of the absorptions of the dithiafulvene protons in the repeating unit with those of the absorptions of the terminal thioamide protons results in the estimation of number-average degree of polymerization. The model compound 3 was prepared according to ref 15 .

\section{Measurements}

${ }^{1} \mathrm{H}$ NMR and IR spectra were recorded on a JEOL JNM-EX270 spectrometer and a Perkin Elmer 1600 spectrometer, respectively. UV-Vis spectra were obtained on a JASCO V-530 spectrophotometer. For cyclic voltammetry studies, thin polymer films were deposited on an indium-tin-oxide (ITO) coated glass electrode by casting from a dimethyl sulfoxide (DMSO) solution. The electrodes were dried under vacuum. Cyclic voltammetry was carried out with a BAS CV-50 W Electrochemi- 


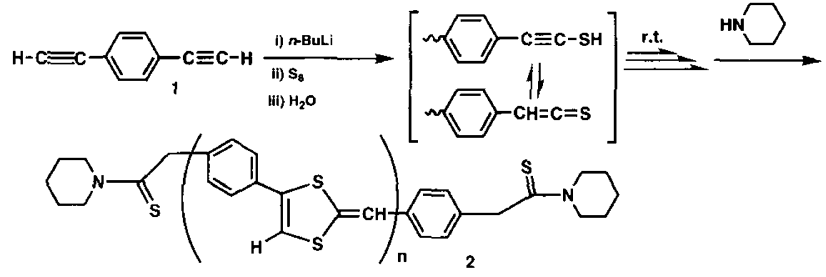

Scheme 1.

cal Analyzer. The cyclic voltammogram of a cast film of $\mathbf{2}$ on an ITO electrode was measured in an acetonitrile solution of $0.1 \mathrm{M}\left[\mathrm{NEt}_{4}\right] \mathrm{BF}_{4}$ at $300 \mathrm{mV} \mathrm{s}^{-1}$. ESR spectra were recorded at X-band frequency with a JEOL JES-RE spectrometer in quartz capillaries of $4 \mathrm{~mm}$ internal diameter. Electrical conductivity was measured at room temperature by two-probe technique using a Keithley model 236 source measure unit.

\section{CT Complex of 2 with TCNQ}

A typical experimental procedure is as follows. An excess amount of TCNQ (10 mg) against repeating unit of 2 was added to a solution of $2(3 \mathrm{mg})$ in DMSO $(0.5 \mathrm{~mL})$, and the mixture was stirred for $15 \mathrm{~min}$ at room temperature. The solution was gradually turned to dark green. After the unreacted TCNQ was filtered off, the solvent was removed under the reduced pressure to obtained a dark green powder.

Yield : $5 \mathrm{mg} .{ }^{1} \mathrm{H} \mathrm{NMR}(\delta, \mathrm{ppm}): 1.2-1.7\left(\mathrm{CH}_{2}\right.$ of piperidine unit), $3.6\left(\mathrm{~N}-\mathrm{CH}_{2}\right.$ of piperidine unit), $4.2(\mathrm{~N}-$ $\mathrm{CH}_{2}$ of piperidine unit), $4.3\left(\mathrm{CH}_{2} \mathrm{C}(=\mathrm{S}) \mathrm{N}<\right), 7.2-8.2$ (aromatic and dithiafulvene of 2 and TCNQ); IR ( $\mathrm{KBr}$, $\left.\mathrm{cm}^{-1}\right) 2201(\mathrm{C} \equiv \mathrm{N}$ stretching of TCNQ), $2179(\mathrm{C} \equiv \mathrm{N}$ stretching of TCNQ), $1576(\mathrm{C}=\mathrm{C}$ vibration of the dithiafulvene unit), 1505 (terminal thioamide group).

\section{RESULTS AND DISCUSSION}

Poly(dithiafulvene) (2) used in this study was prepared by the cycloaddition polymerization of the bis(aldothioketene) derived from 1,4-diethynylbenzene (1) according to the previous our report (Scheme 1). ${ }^{13,14}$ In DMSO, 2 reacted with TCNQ to produce a dark green homogeneous solution. An excess amount of TCNQ against the repeating unit of $\mathbf{2}$ was added to a solution of 2 in DMSO, and the mixture was stirred for $15 \mathrm{~min}$ at room temperature. The polymer solution gradually turned to dark green, which indicated the chargetransfer (CT) complex formation. Due to very low solubility of TCNQ itself in DMSO at room temperature, unreacted TCNQ was remained as precipitate. After the precipitated TCNQ was filtered off, the filtrate was evaporated to obtain a dark green powder. The resulting CT complex was soluble in acetonitrile, DMSO, $N, N$ dimethylformamide (DMF), acetone, and $\mathrm{MeOH}$, partially soluble in THF, and not soluble in $\mathrm{CHCl}_{3}, \mathrm{Et}_{2} \mathrm{O}$, and $\mathrm{H}_{2} \mathrm{O}$. On the contrary, the starting polymer was soluble in DMSO and DMF, and partially soluble in acetone and methanol.

The UV-Vis absorption spectrum of the anion radical of TCNQ exhibits major maxima at 420 and $842 \mathrm{~nm}$. The intensity ratio of the 420 and $842 \mathrm{~nm}$ band is about 0.5

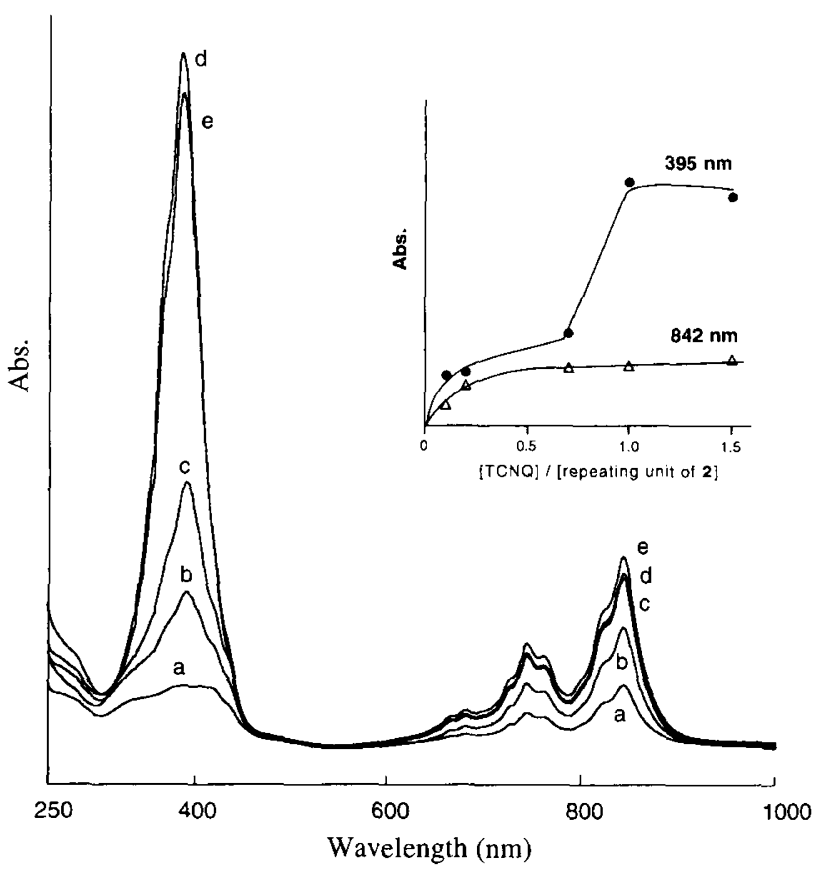

Figure 1. Dependence of the UV-Vis absorption spectrum on the TCNQ feed ratio against the 1,3-dithiafulvene unit of 2 . [TCNQ]/ [repeating unit of 2] $=0.1$ (a), 0.2 (b), 0.7 (c), 1.0 (d), and $1.5(\mathrm{e})$. The inset shows the relative intensity at 393 and $842 \mathrm{~nm} v s$. [TCNQ]/[repeating unit of 2].

for simple salts, ${ }^{16,17}$ and determination of this ratio serves as a useful criterion in characterization of TCNQ derivatives. TCNQ shows a strong absorption at around $395 \mathrm{~nm}$. The UV-Vis absorption spectrum of the CT complex of 2 with TCNQ showed an absorption which was essentially a summation of the band characteristic of TCNQ and the anion-radical. The intensity ratio of the 395 and $842 \mathrm{~nm}$ band was approximately three. Figure 1 shows the dependence of the UV-Vis absorption spectrum on the TCNQ feed ratio against the dithiafulvene unit of 2. At [TCNQ]/[repeating unit of 2] $=0.1$, the absorption spectrum was a typical radical-anion salt of TCNQ. Increased feed ratio of TCNQ resulted in an increase in the absorption at $395 \mathrm{~nm}$. The relative intensities at 395 and $842 \mathrm{~nm}$ were plotted as a function of [TCNQ]/[repeating unit of 2] (Figure 1, inset). The intensity at $842 \mathrm{~nm}$ was saturated below [TCNQ]/[repeating unit of 2] $=0.7$. In the relative intensity at $395 \mathrm{~nm}$, the first saturation curve profile is observed below [TCNQ]/ [repeating unit of 2] $=0.7$. Further increasing the feed ratio of TCNQ beyond [TCNQ]/[repeating unit of 2] = 1.0 did not increase the absorption at $395 \mathrm{~nm}$, which clearly suggested a final formation of a complex containing about 1:1 ratio of dithiafulvene unit to TCNQ. These results indicate that the anion radical of TCNQ was initially formed by the charge-transfer reaction when TCNQ was added to the solution of 2 , and the remaining TCNQ was interacted with 2 as a partially charge-transferred form.

FT-IR spectrum of the sample containing about $1: 1$ ratio of dithiafulvene unit to TCNQ shows both frequency corresponding to $\mathbf{2}$ and TCNQ (See EXPERIMENTAL Section). The degree of charge transfer can be determined by the frequency of the nitrile stretching band of 


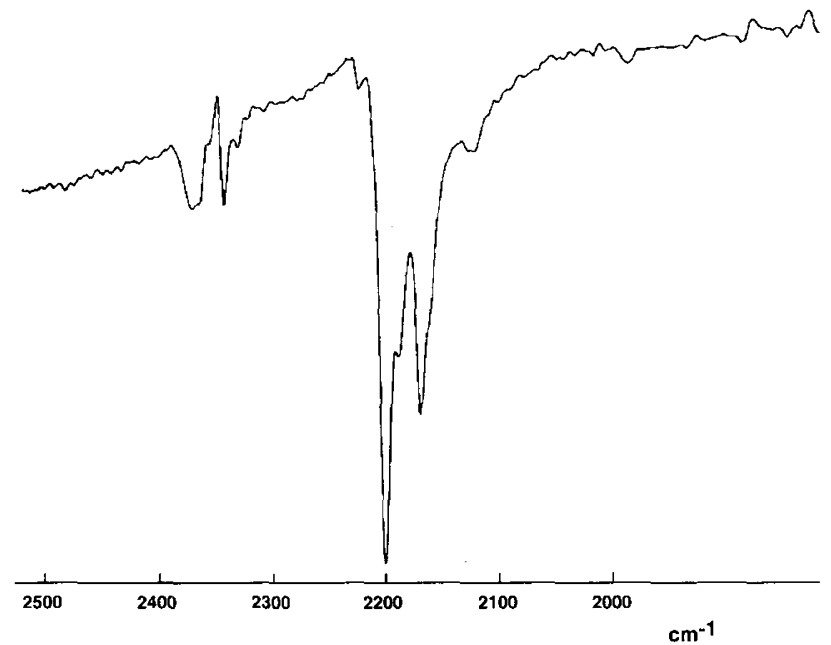

Figure 2. FT-IR spectrum of the CT complex of 2 containing about $1: 1$ ratio of dithiafulvene unit to TCNQ.

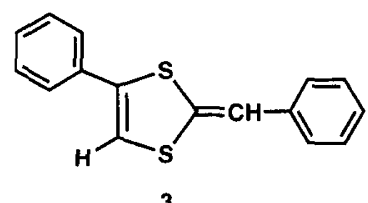

Structure 3.

TCNQ. ${ }^{18,19}$ Neutral TCNQ shows $2227 \mathrm{~cm}^{-1}$ and fully ionic TCNQ is $2180 \mathrm{~cm}^{-1}$. The spectrum of the CT complex of 2 with TCNQ shows a major band in $\mathrm{C} \equiv \mathrm{N}$ stretching region at $2201 \mathrm{~cm}^{-1}$ and a minor band at $2179 \mathrm{~cm}^{-1}$ (Figure 2), indicating two different types of TCNQ molecules in the sample. The latter band was assignable to the anion radical of TCNQ. The major frequency at $2201 \mathrm{~cm}^{-1}$ was shifted with respect to that of the partially charge-transferred TCNQ. The degree of charge transfer was estimated to be $0.62 .^{18}$ This value was almost same as those of most organic CT complexes. ${ }^{18}$ FT-IR spectrum of the sample containing about $3: 1$ ratio of dithiafulvene unit to TCNQ shows a major band in $\mathrm{C} \equiv \mathrm{N}$ stretching region at $2177 \mathrm{~cm}^{-1}$, indicating the anion radical of TCNQ was mainly formed. These observations are consistent with the UV-Vis absorption analysis described above.

Figures 3 and 4 show the dependence of ${ }^{1} \mathrm{H}$ NMR spectrum on the TCNQ feed ratio against 2-benzylidene-4phenyl-1,3-dithiole (3) as a model compound and the dithiafulvene unit of 2 . The ${ }^{1} \mathrm{H}$ NMR spectrum of the model compound 3 shows a peak for the benzylidene proton appeared at $6.7 \mathrm{ppm}$, along with multiplet peaks for the 1,3-dithiole ring proton at $7.2 \mathrm{ppm}$ (Figure 3a). Although the benzylidene proton and the 1,3-dithiole ring proton of 3 disappeared after an excess amount of TCNQ was added to the DMSO- $d_{6}$ solution of 3 (Figure $3 \mathrm{c}$ ), the peaks at 6.7 and $7.2 \mathrm{ppm}$ were still observed when a half equimolar amount of TCNQ was added to the DMSO- $d_{6}$ solution of 3 (Figure $3 b$ ). In addition to these peaks, several new peaks appeared between 6.8 and $7.2 \mathrm{ppm}$, indicating several different types of dithiafulvene molecules in the sample. In the ${ }^{1} \mathrm{H}$ NMR spectrum of the neutral

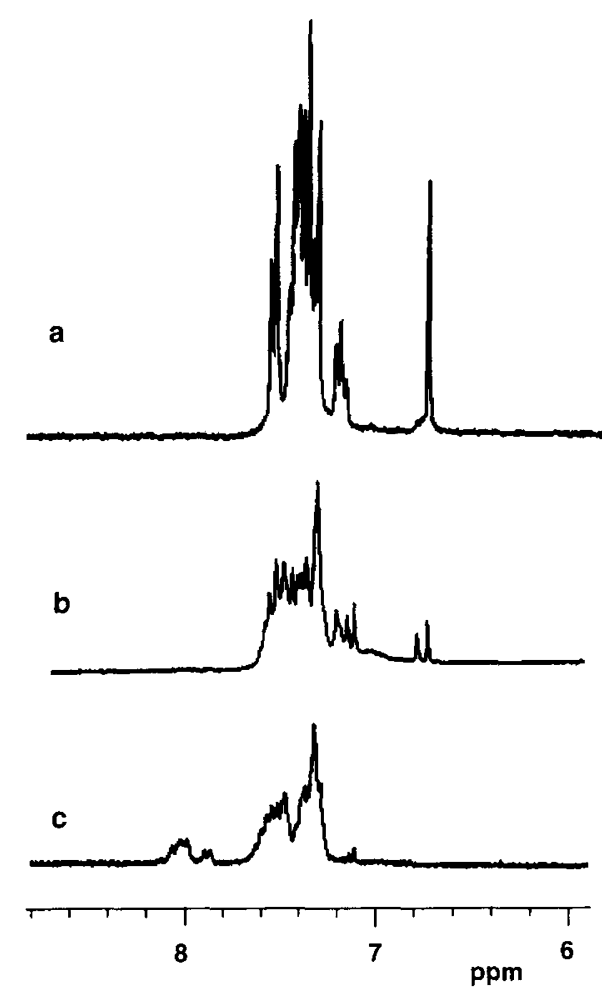

Figure 3. Dependence of ${ }^{1} \mathrm{H}$ NMR spectrum on the TCNQ feed ratio against the model compound $\mathbf{3}$ in DMSO- $d_{6}$. [TCNQ]/[3] $=0$ (a), $0.5(\mathrm{~b})$, and 3 (c).
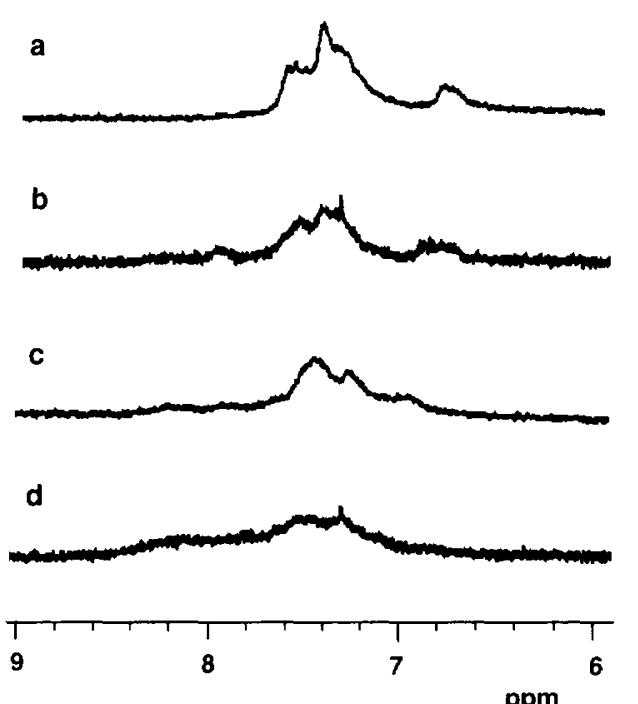

Figure 4. Dependence of ${ }^{1} \mathrm{H}$ NMR spectrum on the TCNQ feed ratio against the dithiafulvene unit of $\mathbf{2}$ in DMSO- $d_{6}$. [TCNQ]/[repeating unit of 2$]=0(\mathrm{a}), 0.1(\mathrm{~b}), 0.3(\mathrm{c})$, and $3(\mathrm{~d})$.

poly(dithiafulvene) 2 (Figure 4a), a broad peak for the benzylidene proton appeared at $6.7 \mathrm{ppm}$. The amount of TCNQ against the repeating unit of 2 to the DMSO- $d_{6}$ solution of 2 was increased, and benzylidene proton at $6.7 \mathrm{ppm}$ was shifted to a lower magnetic field (Figures $4 \mathrm{~b}-4 \mathrm{~d}$ ). Even 0.3 equimolar amount of TCNQ was added to the DMSO- $d_{6}$ solution of $\mathbf{2}$, a broad peak appeared at $6.95 \mathrm{ppm}$ and the peak for the neutral benzylidene proton at $6.7 \mathrm{ppm}$ was hardly observed (Figure 4c). These results indicated that electron density of the 


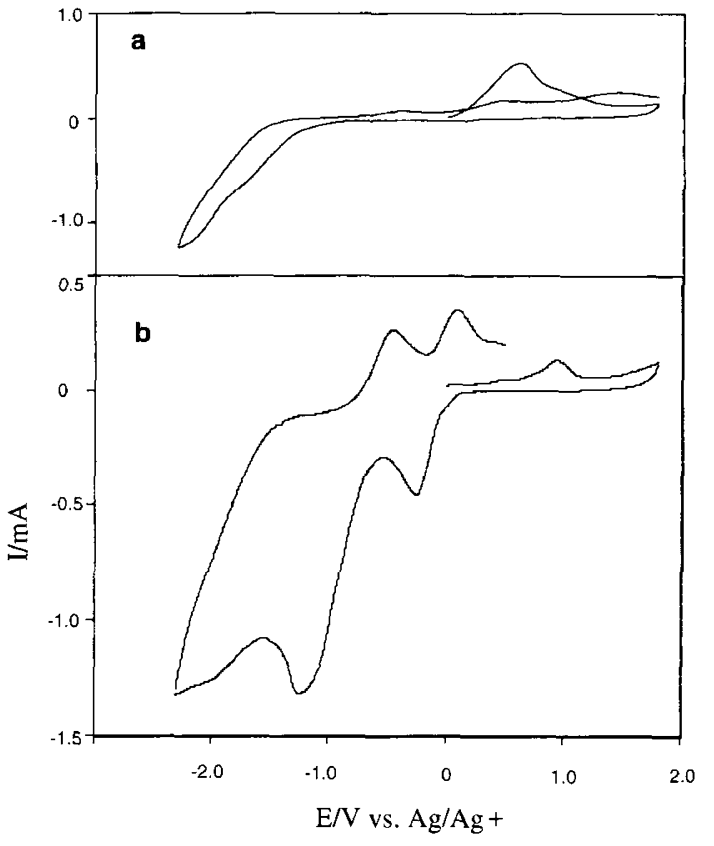

Figure 5. Cyclic voltammogram of 2 (a) and the CT complex of 2 with TCNQ (b) in an acetonitrile solution of $0.1 \mathrm{M}\left[\mathrm{NEt}_{4}\right] \mathrm{BF}_{4}$ at $300 \mathrm{mV} \mathrm{s}^{-1}$.

dithiafulvene units decreased by charge-transfer reaction with TCNQ and the positive charge in the dithiafulvene moieties was effectively delocalized in the $\pi$ conjugated polymer chain.

The ${ }^{1} \mathrm{H}$ NMR spectrum of the neutral poly(dithiafulvene) 2 showed two broad peaks at 4.2 and $3.6 \mathrm{ppm}$ attributed to the terminal thioamide moiety. Comparison of the intensities of the absorptions of the dithiafulvene protons in the repeating unit with those of the absorptions of the terminal thioamide protons resulted in the estimation of number-average degree of polymerization. The ${ }^{1} \mathrm{H}$ NMR spectrum after an excess amount of TCNQ was added to the DMSO- $d_{6}$ solution of 2 showed very broad peak from 7.0 to $8.5 \mathrm{ppm}$ assignable to aromatic and dithiafulvene protons of 2 and TCNQ, along with two broad peaks at 4.2 and $3.6 \mathrm{ppm}$ attributed to the terminal thioamide protons. The peaks for unreacted TCNQ hardly appeared in the ${ }^{1} \mathrm{H}$ NMR spectrum due to the limited solubility of TCNQ in DMSO at room temperature. Comparison of the intensities of the absorptions of the aromatic region of $\mathbf{2}$ and TCNQ protons with those of the absorptions of the terminal thioamide protons showed a formation of a complex containing about $1: 1$ ratio of dithiafulvene unit to TCNQ.

To estimate the redox behavior of the CT complex of $\mathbf{2}$, a cyclic voltammetric measurement was carried out. One oxidation peak was observed at $0.9 \mathrm{~V}$ vs. $\mathrm{Ag} / \mathrm{Ag}^{+}$, which can be assigned to one electron transfer from CT complex of 2 forming neutral TCNQ and the multi-cationic states of 2. The oxidation peak corresponded to the neutral dithiafulvene unit was hardly recognized, i.e., cast film of 2 gave a single-broad oxidation peak at about 0.6 $\mathrm{V}$ vs. $\mathrm{Ag} / \mathrm{Ag}^{+}$. The two reduction peaks at about -0.3 and $-1.0 \mathrm{~V}$ vs. $\mathrm{Ag} / \mathrm{Ag}^{+}$, respectively were assignable to the reduction of neutral TCNQ. ${ }^{20}$ The drastic shift of the oxidation peak for the CT complex of 2 results from an

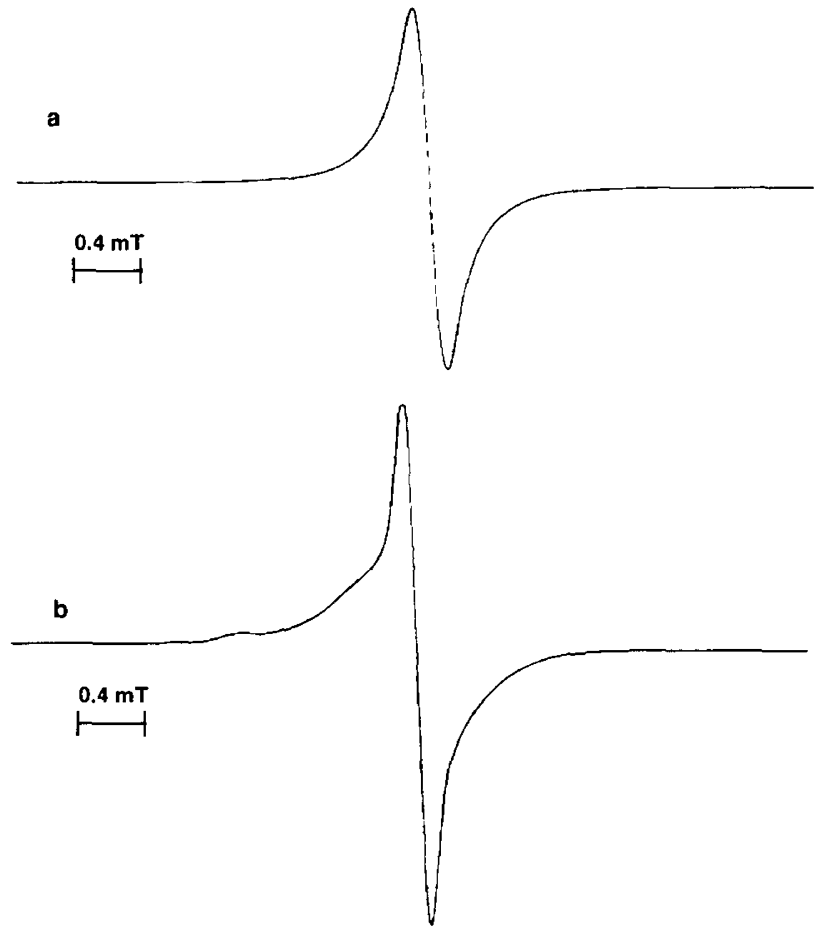

Figure 6. ESR spectra the CT complex of 3 and 2. Both samples contained about $1: 1$ ratio of dithiafulvene unit to TCNQ.

electron withdrawing effect of TCNQ. The cyclic voltammetric analysis also confirmed that dithiafulvene unit in 2 was oxidized by TCNQ and neutral dithiafluvene moiety hardly existed.

ESR spectra were measured for the isolated CT complexes of 2 and 3 diluted with KCl. The ESR spectrum of TCNQ reacting with 3 appeared a narrow singlet signal having $g$ value of 2.003 , indicating existence of a stable anion radical of TCNQ. ${ }^{21,22}$ The ESR spectrum of the CT complex of 2 consists of two types of signals. The $g$ value and the lineshape of a sharp signal was completely same as that of the model CT complex. This sharp signal ( $g=$ 2.003) was asigned to the anion radical of TCNQ. One broad singlet line $(g=2.013)$ was clearly visible at higher modulation level. The $g$ value of the broad signal is almost same as that of the ESR signal of TTF-TCNQ complex. ${ }^{23-25}$ The broad signal may corresponded to the partially charge-transferred TCNQ with 2.

Before doping, 2 exhibits an electrical conductivity of 3 $\times 10^{-7} \mathrm{~S} \mathrm{~cm}^{-1}$ as measured on a cast film from 1,3dimethyl-2-imidazolidinone. When 2 was dissolved in the same solvent with a slightly excess amount of TCNQ against dithiafluvene unit of 2, a black CT complex was obtained. A cast film of the CT complex had a conductivity of $2 \times 10^{-4} \mathrm{~S} \mathrm{~cm}^{-1}$, three orders of magnitude greater than the uncomplexed polymer. Oxidation of 2 with iodine instead of TCNQ raised the electrical conductivity of $1 \times 10^{-3} \mathrm{~S} \mathrm{~cm}^{-1}$.

\section{CONCLUSIONS}

We present the first example of a soluble CT complex of $\pi$-conjugated polymers containing dithiafulvene units in the main chain with an organic acceptor such as TCNQ. The electronic absorption spectra suggested a 


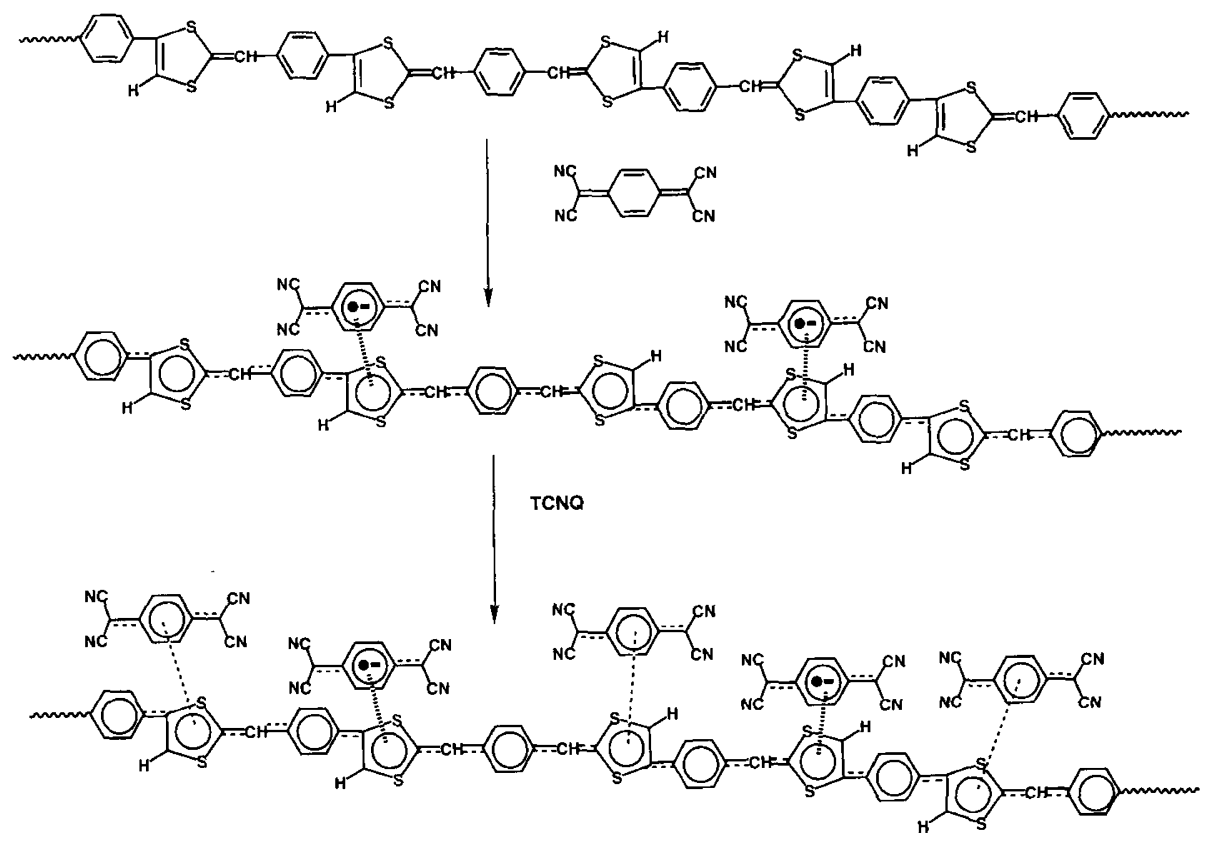

Scheme 2.

formation of the CT complex containing about $1: 1$ ratio of dithiafulvene unit to TCNQ. The UV-Vis absorption and FT-IR analyses indicated that the anion radical of TCNQ was initially formed by the charge-transfer reaction when TCNQ was added to the solution of 2 , and the remaining TCNQ was interacted with 2 as a partially charge-transferred form (Scheme 2). The charge-transfer reaction of dithiafluvene unit of the polymer was supported by the ${ }^{1} \mathrm{H}$ NMR and the cyclic voltammetric measurements. In the ${ }^{1} \mathrm{H}$ NMR spectra, the broad peak attributed to the benzylidene proton of 2 was shifted to a lower magnetic field proportional to the TCNQ feed ratio against the dithiafulvene unit of $\mathbf{2}$. This observation indicated that electron density of the dithiafulvene units decreased by charge-transfer reaction with TCNQ and the positive charge in the dithiafulvene moieties was effectively delocalized in the $\pi$-conjugated polymer chain. The oxidation level of the electron donating $\pi$-conjugated polymer can be controlled by the amount of the acceptor.

Acknowledgment. We would like to thank Dr. A. Yamamoto and Dr. M. Fukushima of Shin-Etsu Chemical Co. for providing the electrical conductivity data. We also thank Dr. Y. Misaki for helpful discussions.

\section{REFERENCES}

1. M. R. Bryce, Chem. Soc. Rev., 20, 335 (1991).

2. T. K Hansen and J. Becher, Adv. Mater., 5, 288 (1993).

3. M. R. Bryce, J. Mater. Chem., 5, 1481 (1995).

4. J. Roncali, J. Mater. Chem., 7, 2307 (1997).

5. M. Adam and K. Müllen, Adv. Mater., 6, 439 (1994).

6. Y. Ueno, Y. Masuyama, and M. Okawara, Chem. Lett., 603 (1975).
7. C. U. Pittman, Jr., Y.-F. Liang, and M. Ueda, Macromolecules, 12, 541 (1979).

8. C. U. Pittman, Jr., M. Narita, and M. Y.-F. Liang, Macromolecules, 9, 360 (1976).

9. W. R. Hertler, J. Org. Chem., 41, 1412 (1976).

10. G. Ko $\beta$ mehl and M. Rohde, Makromol. Chem., 183, 2077 (1982).

11. S. Frenzel, S. Arndt, M. R. Gregorious, and K. Müllen, J. Mater. Chem., 5, 1529 (1995).

12. T. Yamamoto and T. Shimizu, J. Mater. Chem., 7, 1967 (1997).

13. K. Naka, T. Uemura, and Y. Chujo, Macromolecules, 31, 7570 (1998).

14. K. Naka, T. Uemura, and Y. Chujo, Macromolecules, 32, 4641 (1999).

15. R. Raap, Can. J. Chem., 46, 2251 (1968).

16. L. R. Melby, R. J. Harder, W. R. Hertler, W. Mahler, R. E. Benson, and W. E. Mochel, J. Am. Chem. Soc., 84, 3374 (1962).

17. J. P. Gong, I. Kawakami, V. J. Sergeyev, and Y. Osada, Macromolecules, 24, 5246 (1991).

18. J. S. Chappell, A. N. Bloch, W. A. Bryden, M. Maxfield, M. T. O. Poehler, and D. O. Cowan, J. Am. Chem. Soc., 103, 2442 (1981).

19. P. J. Skabara, K. Müllen, M. R. Bryce, J. A. K. Howard, and A. S. Batsanov, J. Mater. Chem., 8, 1719 (1998).

20. W. L. Wallace, C. D. Jaeger, and A. J. Bard, J. Am. Chem. Soc., 101, 4840 (1979).

21. L. E. Bromberg and M. G. Gold, J. Phys. Chem. B, 101, 966 (1997).

22. K. Mizoguchi, H. Togou, E. Tsuchida, and I. Shinihara, Nippon Kagaku Kaishi, 2211 (1975).

23. Y. Tomkiewicz, J. R. Andersen, and A. R. Taranko, Phys. Rev. $B, 17,1579$ (1978).

24. Y. Tomkiewicz and A. R. Taranko, Phys. Rev. B, 16, 1380 (1977).

25. Y. Tomkiewicz, A. R. Taranko, and J. B. Torrance, Phys. Rev. $B, 15,1017$ (1977). 